\title{
Threshold Dynamics of a Stochastic Chemostat Model with Two Nutrients and One Microorganism
}

\author{
Jian Zhang, ${ }^{1}$ Anqi Miao, ${ }^{1}$ and Tongqian Zhang ${ }^{1,2}$ \\ ${ }^{1}$ College of Mathematics and Systems Science, Shandong University of Science and Technology, Qingdao 266590, China \\ ${ }^{2}$ State Key Laboratory of Mining Disaster Prevention and Control Co-Founded by Shandong Province and the Ministry of \\ Science and Technology, Shandong University of Science and Technology, Qingdao 266590, China
}

Correspondence should be addressed to Tongqian Zhang; zhangtongqian@sdust.edu.cn

Received 30 March 2017; Revised 15 July 2017; Accepted 20 August 2017; Published 25 September 2017

Academic Editor: Zhongwei Lin

Copyright (C) 2017 Jian Zhang et al. This is an open access article distributed under the Creative Commons Attribution License, which permits unrestricted use, distribution, and reproduction in any medium, provided the original work is properly cited.

\begin{abstract}
A new stochastic chemostat model with two substitutable nutrients and one microorganism is proposed and investigated. Firstly, for the corresponding deterministic model, the threshold for extinction and permanence of the microorganism is obtained by analyzing the stability of the equilibria. Then, for the stochastic model, the threshold of the stochastic chemostat for extinction and permanence of the microorganism is explored. Difference of the threshold of the deterministic model and the stochastic model shows that a large stochastic disturbance can affect the persistence of the microorganism and is harmful to the cultivation of the microorganism. To illustrate this phenomenon, we give some computer simulations with different intensity of stochastic noise disturbance.
\end{abstract}

\section{Introduction}

Chemostat is commonly used to describe the dynamics of a microbial population in a continuous bioreactor in which microorganisms grow on a substrate and has attracted great interest of many scholars [1-8], since it was first introduced by Monod [9]. A single simple species chemostat model with Michaelis-Menten-Monod functional response was proposed by [9] as follows:

$$
\begin{aligned}
\frac{\mathrm{d} S(t)}{d t} & =D\left(S^{0}-S(t)\right)-\frac{r}{\delta} \frac{S(t) X(t)}{K+S(t)}, \\
\frac{\mathrm{d} X(t)}{d t} & =\frac{r S(t) X(t)}{K+S(t)}-D X(t),
\end{aligned}
$$

where $S(t)$ is the concentration of the nutrient, $X(t)$ is the concentration of the organism, $D$ is the dilution (or washout) rate, $r$ is the maximal growth rate, $K$ is the Michaelis-Menten (or half-saturation) constant with units of concentration, and $\delta$ is a "yield" constant reflecting the conversion of nutrient to organism.

However, experimental results have indicated that the microorganisms depend on a variety of nutrition substances such as carbon, nitrogen, energy, growth factors, inorganic salts, and water. Then the model of microorganisms species growth in the chemostat on two nutrients is considered by [10-14]. A model of single-species growth in the chemostat on two substitutable resources with Michaelis-Menten-Monod functional response was proposed by [14] as follows:

$$
\begin{aligned}
& \frac{\mathrm{d} S_{1}(t)}{d t}=D\left(S_{1}^{0}-S_{1}(t)\right)-\frac{r_{1} S_{1}(t) X(t)}{K_{1}+S_{1}(t)}, \\
& \frac{\mathrm{d} S_{2}(t)}{d t}=D\left(S_{2}^{0}-S_{2}(t)\right)-\frac{r_{2} S_{2}(t) X(t)}{K_{2}+S_{2}(t)}, \\
& \frac{\mathrm{d} X(t)}{d t}=\frac{r_{1} S_{1}(t) X(t)}{K_{1}+S_{1}(t)}+\frac{r_{2} S_{2}(t) X(t)}{K_{2}+S_{2}(t)}-D X(t) .
\end{aligned}
$$

However, it is now well known that stochastic noise is widely present in biological systems and so on [15-33] and microorganisms are inevitably influenced by some random factors in the process of cultivation. To better understand the dynamic behavior of the chemostat, a host of scholars proposed a slice of stochastic chemostat models and studied the effect of the random noise on the dynamic behavior of the stochastic models. As an example, Imhof and Walcher [34] 
proposed a stochastic chemostat model for a single microorganism species consuming a single nutrient. They found that random effects may lead to extinction in scenarios where the deterministic model predicts persistence. Recently, $\mathrm{Xu}$ and Yuan [35] established a stochastic chemostat model in which the maximal growth rate is influenced by the white noise in environment as follows:

$$
\begin{aligned}
\mathrm{d} S(t)= & \left(D\left(S^{0}-S(t)\right)-\frac{m S(t) X(t)}{a+S(t)}\right) \mathrm{d} t \\
& -\frac{\alpha S(t) X(t) \mathrm{d} B(t)}{a+S(t)}, \\
\mathrm{d} X(t)= & \left(\frac{m S(t) X(t)}{a+S(t)}-D X(t)\right) \mathrm{d} t \\
& +\frac{\alpha S(t) X(t) \mathrm{d} B(t)}{a+S(t)} .
\end{aligned}
$$

They got an analogue break-even concentration involving the white noise which can determine the exclusion and persistence of the microorganism. And more stochastic chemostat models can be found in [36-39].

Motivated by the papers mentioned above, in this paper, we further consider a model of single-species growth in the chemostat on two supplementary resources with MichaelisMenten-Monod functional response and environmental noise. We assume that the maximal growth rate $r_{i}(i=1,2)$ is perturbed by white noises so that

$$
r_{i} \longrightarrow r_{i}+\sigma_{i} \dot{B}_{i}(t)
$$

where $B_{i}(t)$ is a standard Brownian motion with intensity $\sigma_{i}>$ 0 . Then the resultant model takes the following form:

$$
\begin{aligned}
\mathrm{d} S_{1}(t)= & \left(D\left(S_{1}^{0}-S_{1}(t)\right)-\frac{r_{1} S_{1}(t) X(t)}{K_{1}+S_{1}(t)}\right) \mathrm{d} t \\
& -\frac{\sigma_{1} S_{1}(t) X(t) \mathrm{d} B_{1}(t)}{K_{1}+S_{1}(t)}, \\
\mathrm{d} S_{2}(t)= & \left(D\left(S_{2}^{0}-S_{2}(t)\right)-\frac{r_{2} S_{2}(t) X(t)}{K_{2}+S_{2}(t)}\right) \mathrm{d} t \\
& -\frac{\sigma_{2} S_{2}(t) X(t) \mathrm{d} B_{2}(t)}{K_{2}+S_{2}(t)}, \\
\mathrm{d} X(t)= & \left(\frac{r_{1} S_{1}(t) X(t)}{\left.K_{1}+S_{1}(t)+\frac{r_{2} S_{2}(t) X(t)}{K_{2}+S_{2}(t)}-D X(t)\right) \mathrm{d} t}\right. \\
& +\frac{\sigma_{1} S_{1}(t) X(t) \mathrm{d} B_{1}(t)}{K_{1}+S_{1}(t)} \\
& +\frac{\sigma_{2} S_{2}(t) X(t) \mathrm{d} B_{2}(t)}{K_{2}+S_{2}(t)} .
\end{aligned}
$$

Our main objective in the rest of this paper is to investigate the threshold dynamics of stochastic chemostat model (5) and explore the conditions under which microorganisms will die out or exist.

\section{Preliminaries}

In this section, we will give some notations, definitions, and lemmas which will be used for analyzing our main results. To this end, throughout this paper, we let $\left(\Omega, \mathscr{F},\{\mathscr{F}\}_{t \geq 0}, \mathscr{P}\right)$ be a complete probability space with a filtration $\left\{\mathscr{F}_{t}\right\}_{t \geq 0}$ satisfying the usual conditions: it is increasing and right continuous while $\mathscr{F}_{0}$ contains all $\mathscr{P}$-null sets; we use $B(t)$ to represent a scalar Brownian motion defined on the complete probability space $\Omega$; also let $R_{+}^{2}=\left\{x_{i}>0, i=1,2\right\}$. If for an integrable function $f$ on $[0,+\infty)$, define

$$
\langle f(t)\rangle=\frac{1}{t} \int_{0}^{t} f(\theta) \mathrm{d} \theta .
$$

Then we have the following.

Definition 1. For system (5),

(i) the microorganism $X(t)$ is said to be extinctive if $\lim _{t \rightarrow+\infty} X(t)=0$,

(ii) the microorganism $X(t)$ is said to be permanent in mean if there exists a positive constant $\lambda$ such that $\liminf \operatorname{int+\infty }_{t \rightarrow}\langle X(t)\rangle \geq \lambda$.

Then, one can show the following lemmas.

Lemma 2. The solution $\left(S_{1}(t), S_{2}(t), X(t)\right)$ of model (2) or (5) with the initial condition $\left(S_{1}(0), S_{2}(0), X(0)\right) \in R_{+}^{3}$ is ultimately bounded; that is,

$$
\begin{aligned}
& \limsup _{t \rightarrow \infty} S_{1}(t) \leq S_{1}^{0}, \\
& \limsup _{t \rightarrow \infty} S_{2}(t) \leq S_{2}^{0}, \\
& \limsup _{t \rightarrow \infty} M(t) \leq S_{1}^{0}+S_{2}^{0},
\end{aligned}
$$

where $M(t)=S_{1}(t)+S_{2}(t)+X(t)$.

Proof. Letting $M(t)=S_{1}(t)+S_{2}(t)+X(t)$, from system (2) or system (5), we have

$$
\frac{\mathrm{d} M(t)}{\mathrm{d} t}=D\left(S_{1}^{0}+S_{2}^{0}\right)-D M(t)
$$

This implies that

$$
\lim _{t \rightarrow+\infty} M(t)=S_{1}^{0}+S_{2}^{0}
$$

Thus, we have

$$
\begin{aligned}
& \limsup _{t \rightarrow \infty} S_{1}(t) \leq S_{1}^{0}, \\
& \limsup _{t \rightarrow \infty} S_{2}(t) \leq S_{2}^{0}, \\
& \limsup _{t \rightarrow \infty} M(t)=S_{1}^{0}+S_{2}^{0} .
\end{aligned}
$$

This completes the proof of Lemma 2. 
By Lemma 2 and the strong law of large numbers for martingales [40], we can obtain the following lemma.

Lemma 3. Letting $\left(S_{1}(t), S_{2}(t), X(t)\right)$ be a solution of system (5) with initial value $\left(S_{1}(0), S_{2}(0), X(0)\right) \in R_{+}^{3}$, then

$$
\begin{aligned}
& \lim _{t \rightarrow+\infty} \frac{1}{t} \int_{0}^{t} \frac{\sigma_{1} S_{1}(\tau)}{K_{1}+S_{1}(\tau)} \mathrm{d} B(\tau)=0, \\
& \lim _{t \rightarrow+\infty} \frac{1}{t} \int_{0}^{t} \frac{\sigma_{2} S_{2}(\tau)}{K_{2}+S_{2}(\tau)} \mathrm{d} B(\tau)=0 .
\end{aligned}
$$

\section{Dynamics of Deterministic System (2)}

In this section, we will focus on the deterministic system (2). It is easy to see that the equilibria point of (2) satisfy

$$
\begin{array}{r}
D\left(S_{1}^{0}-S_{1}(t)\right)-\frac{r_{1} S_{1}(t) X(t)}{K_{1}+S_{1}(t)}=0, \\
D\left(S_{2}^{0}-S_{2}(t)\right)-\frac{r_{2} S_{2}(t) X(t)}{K_{2}+S_{2}(t)}=0, \\
\frac{r_{1} S_{1}(t) X(t)}{K_{1}+S_{1}(t)}+\frac{r_{2} S_{2}(t) X(t)}{K_{2}+S_{2}(t)}-D X(t)=0,
\end{array}
$$

and, obviously, model (2) has a microorganism extinction equilibrium $E_{0}\left(S_{1}^{0}, S_{2}^{0}, 0\right)$. Let $E^{*}\left(S_{1}^{*}, S_{2}^{*}, X^{*}\right)$ be the coexistence equilibrium of model (2), which satisfies

$$
f\left(S_{2}\right)=: a\left(S_{2}\right)^{3}+b\left(S_{2}\right)^{2}+c S_{2}+d=0,
$$

where

$$
\begin{aligned}
a= & \left(r_{2}-D\right)\left(r_{1}+r_{2}-D\right), \\
b= & r_{1} S_{2}^{0} D+D S_{1}^{0} r_{2}-2 r_{1} K_{2} D-r_{2}^{2} S_{1}^{0}+r_{1} K_{2} r_{2}-S_{2}^{0} r_{2}^{2} \\
& -r_{2}^{2} K_{1}+2 D^{2} K_{2}-S_{2}^{0} D^{2}-S_{1}^{0} r_{2} r_{1}-2 r_{2} D K_{2} \\
& +2 S_{2}^{0} r_{2} D+D K_{1} r_{2}-r_{1} S_{2}^{0} r_{2}, \\
c= & -2 S_{2}^{0} D^{2} K_{2}+r_{2} K_{1} D K_{2}-K_{2}^{2} r_{1} D+S_{1}^{0} r_{2} D K_{2} \\
& -K_{2} S_{2}^{0} r_{1} r_{2}+2 D S_{2}^{0} K_{2} r_{1}+D^{2} K_{2}^{2}-S_{1}^{0} r_{2} r_{1} K_{2} \\
& +2 S_{2}^{0} r_{2} D K_{2}, \\
d= & K_{2}^{2} S_{2}^{0} D\left(r_{1}-D\right) .
\end{aligned}
$$

Then we have that

$$
\begin{aligned}
& f(0)=K_{2}^{2} S_{2}^{0} D\left(r_{1}-D\right), \\
& f\left(S_{2}^{0}\right)=D S_{1}^{0} r_{2}\left(S_{2}^{0}\right)^{2}-r_{2}^{2} S_{1}^{0}\left(S_{2}^{0}\right)^{2}-r_{2}^{2} K_{1}\left(S_{2}^{0}\right)^{2} \\
& \quad-S_{1}^{0} r_{2} r_{1}\left(S_{2}^{0}\right)^{2}+D K_{1} r_{2}\left(S_{2}^{0}\right)^{2}+r_{2} K_{1} D K_{2} S_{2}^{0} \\
& \quad+S_{1}^{0} r_{2} D K_{2} S_{2}^{0}-S_{1}^{0} r_{2} r_{1} K_{2} S_{2}^{0} \\
& \quad=r_{2} S_{2}^{0}\left(D\left(S_{1}^{0}+K_{1}\right)\left(S_{2}^{0}+K_{2}\right)-S_{1}^{0} r_{1}\left(K_{2}+S_{2}^{0}\right)\right. \\
& \left.\quad-r_{2} S_{2}^{0}\left(S_{1}^{0}+K_{1}\right)\right) .
\end{aligned}
$$

Denote

$$
\mathscr{R}=\frac{1}{D}\left(\frac{1}{\delta_{1}}+\frac{1}{\delta_{2}}\right),
$$

where $\delta_{1}=\left(K_{1}+S_{1}^{0}\right) / r_{1} S_{1}^{0}, \delta_{2}=\left(K_{2}+S_{2}^{0}\right) / r_{2} S_{2}^{0}$.

Obviously, If $\mathscr{R}>1$, we have $f\left(S_{2}^{0}\right)<0$. If $r_{1}>D$, we have $f(0)>0$. Thus, equation has one positive $\operatorname{root} S_{2}$ at least, and $S_{2} \in\left(0, S_{2}^{0}\right)$.

From the second equation of (12), one gets

$$
X=\frac{D\left(S_{2}^{0}-S_{2}\right)\left(K_{2}+S_{2}\right)}{r_{2} S_{2}} .
$$

Substituting (17) into the first equation of (12), we have

$$
\begin{aligned}
r_{2} S_{2} S_{1}^{2} & +\left(r_{1}\left(S_{2}^{0}-S_{2}\right)\left(K_{2}+S_{2}\right)-r_{2}\left(S_{1}^{0}-K_{1}\right) S_{2}\right) S_{1} \\
& -r_{2} S_{2} S_{1}^{0} K_{1}=0 .
\end{aligned}
$$

Let

$$
\begin{aligned}
g\left(S_{1}\right) & \\
= & r_{2} S_{2} S_{1}^{2} \\
& +\left(r_{1}\left(S_{2}^{0}-S_{2}\right)\left(K_{2}+S_{2}\right)-r_{2}\left(S_{1}^{0}-K_{1}\right) S_{2}\right) S_{1} \\
& -r_{2} S_{2} S_{1}^{0} K_{1} .
\end{aligned}
$$

It is easy to see that

$$
\begin{aligned}
g(0) & =-r_{2} S_{2} S_{1}^{0} K_{1}<0, \\
g\left(S_{1}^{0}\right) & =r_{1} S_{1}^{0}\left(S_{2}^{0}-S_{2}\right)\left(K_{2}+S_{2}\right)>0 .
\end{aligned}
$$

Thus, (18) has one positive root $S_{1}$ at least, and $S_{1} \in\left(0, S_{1}^{0}\right)$.

From the third equation of (12), we have $0<X<S_{1}^{0}+S_{2}^{0}$. Then we have the following theorem.

Theorem 4. If $r_{1}>D$ and $\mathscr{R}>1$, then system (2) has unique positive equilibrium $E^{*}$.

Regarding the stability of these equilibria, we have the following theorem.

Theorem 5. Then for system (2), one has the following.

(i) If $\mathscr{R}<1$, microorganism extinction equilibrium $E_{0}$ is locally stable; if $\mathscr{R}>1$ it is unstable.

(ii) If $r_{1}>D$ and $\mathscr{R}>1$, the coexistence equilibrium $E^{*}$ is locally stable.

Proof. Linearizing the system at the equilibrium $E\left(S_{1}^{\circ}, S_{2}^{\circ}, X^{\circ}\right)$ gives the Jacobian

$$
J=\left(\begin{array}{ccc}
-D-a_{1} & 0 & -a_{4} \\
0 & -D-a_{2} & -a_{3} \\
a_{1} & a_{2} & a_{3}+a_{4}-D
\end{array}\right),
$$


where

$$
\begin{aligned}
& a_{1}=\frac{K_{1} r_{1} X^{\circ}}{\left(K_{1}+S_{1}^{\circ}\right)^{2}}, \\
& a_{2}=\frac{K_{2} r_{2} X^{\circ}}{\left(K_{2}+S_{2}^{\circ}\right)^{2}}, \\
& a_{3}=\frac{r_{2} S_{2}^{\circ}}{K_{2}+S_{2}^{\circ}}, \\
& a_{4}=\frac{r_{1} S_{1}^{\circ}}{K_{1}+S_{1}^{\circ}} .
\end{aligned}
$$

The characteristic equation gives

$$
(\lambda+D)\left(\lambda^{2}+A \lambda+B\right)=0,
$$

where

$$
\begin{aligned}
A= & 2 D+a_{1}+a_{2}-a_{3}-a_{4}, \\
B= & D^{2}+a_{1} D+a_{2} D+a_{1} a_{2}-a_{3} D-a_{4} D-a_{1} a_{3} \\
& -a_{2} a_{4} .
\end{aligned}
$$

Obviously, we have, at $E_{0}$,

$$
\begin{aligned}
& a_{1}=0, \\
& a_{2}=0, \\
& a_{3}=\frac{r_{2} S_{2}^{0}}{K_{2}+S_{2}^{0}}, \\
& a_{4}=\frac{r_{1} S_{1}^{0}}{K_{1}+S_{1}^{0}} .
\end{aligned}
$$

Then we have

$$
\begin{aligned}
& A=2 D-a_{3}-a_{4}, \\
& B=D\left(D-\left(a_{3}+a_{4}\right)\right),
\end{aligned}
$$

and thus if $\mathscr{R}<1$, all the eigenvalues of (23) have negative real part; then, by the stability theory, $E_{0}$ is stable.

And, at $E^{*}$, we have

$$
\begin{aligned}
A= & 2 D+a_{1}+a_{2}-a_{3}-a_{4}=D+a_{1}+a_{2}>0, \\
B= & D^{2}+a_{1} D+a_{2} D+a_{1} a_{2}-a_{3} D-a_{4} D-a_{1} a_{3} \\
& -a_{2} a_{4}=a_{1} a_{2}+a_{1}\left(D-a_{3}\right)+a_{2}\left(D-a_{4}\right)>0 ;
\end{aligned}
$$

here $a_{3}+a_{4}=D$ is used. Then all the eigenvalues of (23) have negative real part; thus, by the stability theory, the diseases equilibrium is stable as long as it exists.

\section{Dynamics of Stochastic System (5)}

4.1. Extinction. In this section, we explore the conditions leading to the extinction of the two infectious diseases. Denote

$$
\begin{aligned}
\mathscr{R}^{*}= & \mathscr{R} \\
& -\frac{1}{D}\left(\frac{\sigma_{1}^{2}}{2}\left(\frac{S_{1}^{0}}{K_{1}+S_{1}^{0}}\right)^{2}-\frac{\sigma_{2}^{2}}{2}\left(\frac{S_{2}^{0}}{K_{2}+S_{2}^{0}}\right)^{2}\right),
\end{aligned}
$$

where $\mathscr{R}$ is introduced in (16). Then we have the following.

Theorem 6. For system (5), if one of the following holds,

(i) $\sigma_{1}^{2}>\delta_{1}, \sigma_{2}^{2}>\delta_{2}$, and $r_{1}^{2} / 2 \sigma_{1}^{2}+r_{2}^{2} / 2 \sigma_{2}^{2}<D$,

(ii) $\sigma_{1}^{2}>\delta_{1}, \sigma_{2}^{2}<\delta_{2}$, and $-D+r_{1}^{2} / 2 \sigma_{1}^{2}+r_{2} S_{2}^{0} /\left(K_{2}+S_{2}^{0}\right)-$ $\left(\sigma_{2}^{2} / 2\right)\left(S_{2}^{0} /\left(K_{2}+S_{2}^{0}\right)\right)^{2}<0$,

(iii) $\sigma_{1}^{2}<\delta_{1}, \sigma_{2}^{2}>\delta_{2}$, and $-D+r_{2}^{2} / 2 \sigma_{2}^{2}+r_{1} S_{1}^{0} /\left(K_{1}+S_{1}^{0}\right)-$ $\left(\sigma_{1}^{2} / 2\right)\left(S_{1}^{0} /\left(K_{1}+S_{1}^{0}\right)\right)^{2}<0$,

(iv) $\sigma_{1}^{2}<\delta_{1}, \sigma_{2}^{2}<\delta_{2}$, and $\mathscr{R}^{*}<1$,

then the microorganism $X(t)$ of system (5) goes to extinction almost surely. Moreover,

$$
\begin{aligned}
& \lim _{t \rightarrow+\infty} S_{1}(t)=S_{1}^{0}, \\
& \lim _{t \rightarrow+\infty} S_{2}(t)=S_{2}^{0},
\end{aligned}
$$

almost surely.

Proof. Let $\left(S_{1}(t), S_{2}(t), X(t)\right)$ be a solution of system (5) with initial value $\left(S_{1}(0), S_{2}(0), X(0)\right) \in R_{+}^{3}$. Applying Itô's formula to system (5) results in

$\mathrm{d} \ln X(t)$

$$
\begin{aligned}
= & \left(r_{1} \phi_{1}(t)+r_{2} \phi_{2}(t)-D-\frac{\sigma_{1}^{2}}{2} \phi_{1}^{2}(t)-\frac{\sigma_{2}^{2}}{2} \phi_{2}^{2}(t)\right) \mathrm{d} t \\
& +\frac{\sigma_{1} S_{1}(t) \mathrm{d} B(t)}{K_{1}+S_{1}(t)}+\frac{\sigma_{2} S_{2}(t) \mathrm{d} B(t)}{K_{2}+S_{2}(t)},
\end{aligned}
$$

where $\phi_{1}(t)=S_{1}(t) X(t) /\left(K_{1}+S_{1}(t)\right), \phi_{2}(t)=S_{2}(t) X(t) /\left(K_{2}+\right.$ $\left.S_{2}(t)\right)$.

Integrating both sides of (30) from 0 to $t$ gives

$\ln X(t)$

$$
\begin{aligned}
= & \int_{0}^{t}\left(r_{1} \phi_{1}(\tau)+r_{2} \phi_{2}(\tau)-\frac{\sigma_{1}^{2}}{2} \phi_{1}^{2}(\tau)-\frac{\sigma_{2}^{2}}{2} \phi_{2}^{2}(\tau)\right) \mathrm{d} \tau \\
& -D t+M_{1}(t)+M_{2}(t)+\ln X(0),
\end{aligned}
$$

where

$$
\begin{aligned}
& M_{1}(t)=\int_{0}^{t} \frac{\sigma_{1} S_{1}(t) \mathrm{d} B(\tau)}{K_{1}+S_{1}(t)}, \\
& M_{2}(t)=\int_{0}^{t} \frac{\sigma_{2} S_{2}(t) \mathrm{d} B(\tau)}{K_{2}+S_{2}(t)},
\end{aligned}
$$


known as the local continuous martingale, and $M(0)=$ 0 . Obviously, we need to estimate the maximum value of $r_{1} \phi_{1}(t)+r_{2} \phi_{2}(t)-\left(\sigma_{1}^{2} / 2\right) \phi_{1}^{2}(t)-\left(\sigma_{2}^{2} / 2\right) \phi_{2}^{2}(t)$.

Let us consider quadratic function

$$
g(z)=a z-\frac{\sigma^{2}}{2} z^{2}, \quad z \in\left[0, \frac{b}{K+b}\right], b>0 .
$$

It is easy to verify that when $\sigma^{2}>\delta=(K+b) / a b, g(z)$ reaches its maximum value $g_{\max }=a^{2} / 2 \sigma^{2}$ at $z=a / \sigma^{2}$; and when $\sigma^{2}<\delta, g(z)$ achieve its maximum value $g_{\max }=\delta_{3}=a b /(K+$ $b)-\left(\sigma^{2} / 2\right)(b /(K+b))^{2}$ at $z=b /(K+b)$. Then, in (31), we have four cases to be discussed, depending on whether $\sigma_{1}^{2}>\delta_{1}$ or $\sigma_{2}^{2}>\delta_{2}$, which are as follows: Case 1: $\sigma_{1}^{2}>\delta_{1}, \sigma_{2}^{2}>\delta_{2}$; Case 2: $\sigma_{1}^{2}>\delta_{1}, \sigma_{2}^{2}<\delta_{2}$; Case 3: $\sigma_{1}^{2}<\delta_{1}, \sigma_{2}^{2}>\delta_{2}$; and Case 4: $\sigma_{1}^{2}<\delta_{1}, \sigma_{2}^{2}<\delta_{2}$.

For Case 1 , since $\sigma_{1}^{2}>\delta_{1}, \sigma_{2}^{2}>\delta_{2}$, then $r_{1} \phi_{1}(t)+$ $r_{2} \phi_{2}(t)-\left(\sigma_{1}^{2} / 2\right) \phi_{1}^{2}(t)-\left(\sigma_{2}^{2} / 2\right) \phi_{2}^{2}(t)$ achieve the maximum value $r_{1}^{2} / 2 \sigma_{1}^{2}+r_{2}^{2} / 2 \sigma_{2}^{2}$. Then we can easily see from (31) that

$$
\begin{aligned}
\ln X(t) \leq & \left(\frac{r_{1}^{2}}{2 \sigma_{1}^{2}}+\frac{r_{2}^{2}}{2 \sigma_{2}^{2}}\right) t-D t+M_{1}(t)+M_{2}(t) \\
& +\ln X(0) .
\end{aligned}
$$

Dividing both sides of (34) by $t>0$, we have

$$
\frac{\ln X(t)}{t} \leq-\left(D-\frac{r_{1}^{2}}{2 \sigma_{1}^{2}}-\frac{r_{2}^{2}}{2 \sigma_{2}^{2}}\right)+\frac{M(t)}{t}+\frac{\ln X(0)}{t}
$$

and, by Lemma 3, we have

$$
\lim _{t \rightarrow+\infty} \frac{M(t)}{t}=0
$$

Then, taking the limit superior on both sides of (35) leads to

$$
\limsup _{t \rightarrow+\infty} \frac{\ln X(t)}{t} \leq-\left(D-\frac{r_{1}^{2}}{2 \sigma_{1}^{2}}-\frac{r_{2}^{2}}{2 \sigma_{2}^{2}}\right)<0,
$$

which implies $\lim _{t \rightarrow+\infty} X(t)=0$, and here $r_{1}^{2} / 2 \sigma_{1}^{2}+r_{2}^{2} / 2 \sigma_{2}^{2}<$ $D$ is used.

Case 2. $\sigma_{1}^{2}>\delta_{1}=\left(K+S_{1}^{0}\right) / r_{1} S_{1}^{0}, \sigma_{2}^{2}<\delta_{2}=\left(K+S_{2}^{0}\right) / r_{2} S_{2}^{0}$.

In this case, we can easily see from (31) that

$$
\begin{aligned}
\ln X(t) \leq & \left(\frac{r_{1}^{2}}{2 \sigma_{1}^{2}}+\frac{r_{2} S_{2}^{0}}{K_{2}+S_{2}^{0}}-\frac{\sigma_{2}^{2}}{2}\left(\frac{S_{2}^{0}}{K_{2}+S_{2}^{0}}\right)^{2}\right) t \\
& -D t+M_{1}(t)+M_{2}(t)+\ln X(0) .
\end{aligned}
$$

Dividing both sides of (38) by $t>0$, we have

$$
\begin{aligned}
& \frac{\ln X(t)}{t} \\
& \leq-\left(D-\frac{r_{1}^{2}}{2 \sigma_{1}^{2}}-\frac{r_{2} S_{2}^{0}}{K_{2}+S_{2}^{0}}+\frac{\sigma_{2}^{2}}{2}\left(\frac{S_{2}^{0}}{K_{2}+S_{2}^{0}}\right)^{2}\right) \\
& \quad+\frac{M(t)}{t}+\frac{\ln X(0)}{t}
\end{aligned}
$$

and, by Lemma 3, we have

$$
\lim _{t \rightarrow+\infty} \frac{M(t)}{t}=0
$$

Then, taking the limit superior on both sides of (38) leads to

$$
\begin{aligned}
& \limsup _{t \rightarrow+\infty} \frac{\ln X(t)}{t} \\
& \quad \leq-\left(D-\frac{r_{1}^{2}}{2 \sigma_{1}^{2}}-\frac{r_{2} S_{2}^{0}}{K_{2}+S_{2}^{0}}+\frac{\sigma_{2}^{2}}{2}\left(\frac{S_{2}^{0}}{K_{2}+S_{2}^{0}}\right)^{2}\right) \\
& \quad<0
\end{aligned}
$$

which implies $\lim _{t \rightarrow+\infty} X(t)=0$.

The same discussion can be used in Case 3; here we omit it.

Next, we consider Case 4: $\sigma_{1}^{2}<\delta_{1}=\left(K+S_{1}^{0}\right) / r_{1} S_{1}^{0}, \sigma_{2}^{2}<$ $\delta_{2}=\left(K+S_{2}^{0}\right) / r_{2} S_{2}^{0}$. From (31), we have

$$
\begin{aligned}
\ln X(t) & \leq\left(\frac{r_{1} S_{1}^{0}}{K_{1}+S_{1}^{0}}-\frac{\sigma_{1}^{2}}{2}\left(\frac{S_{1}^{0}}{K_{1}+S_{1}^{0}}\right)^{2}+\frac{r_{2} S_{2}^{0}}{K_{2}+S_{2}^{0}}\right. \\
& \left.-\frac{\sigma_{2}^{2}}{2}\left(\frac{S_{2}^{0}}{K_{2}+S_{2}^{0}}\right)^{2}\right)-D t+M_{1}(t)+M_{2}(t) \\
& +\ln X(0) .
\end{aligned}
$$

Dividing both sides of (42) by $t>0$, we have

$$
\begin{aligned}
& \frac{\ln X(t)}{t} \leq-\left(D-\frac{r_{1} S_{1}^{0}}{K_{1}+S_{1}^{0}}+\frac{\sigma_{1}^{2}}{2}\left(\frac{S_{1}^{0}}{K_{1}+S_{1}^{0}}\right)^{2}\right. \\
& \left.-\frac{r_{2} S_{2}^{0}}{K_{2}+S_{2}^{0}}+\frac{\sigma_{2}^{2}}{2}\left(\frac{S_{2}^{0}}{K_{2}+S_{2}^{0}}\right)^{2}\right)+\frac{M(t)}{t} \\
& +\frac{\ln X(0)}{t}
\end{aligned}
$$

and, by Lemma 3, we have

$$
\lim _{t \rightarrow+\infty} \frac{M(t)}{t}=0
$$

Then, taking the limit superior on both sides of (43) leads to

$$
\begin{aligned}
& \limsup _{t \rightarrow+\infty} \frac{\ln X(t)}{t} \leq-\left(D-\frac{r_{1} S_{1}^{0}}{K_{1}+S_{1}^{0}}\right. \\
& \left.\quad+\frac{\sigma_{1}^{2}}{2}\left(\frac{S_{1}^{0}}{K_{1}+S_{1}^{0}}\right)^{2}-\frac{r_{2} S_{2}^{0}}{K_{2}+S_{2}^{0}}+\frac{\sigma_{2}^{2}}{2}\left(\frac{S_{2}^{0}}{K_{2}+S_{2}^{0}}\right)^{2}\right) \\
& \quad<0
\end{aligned}
$$


Next, we prove the last conclusion. Given $0<\varepsilon \ll 1$, since $\lim _{t \rightarrow+\infty} X(t)=0$, we have $0<X(t)<\varepsilon$ for $t$ large enough. By the first equation of system (5), we have

$$
\begin{aligned}
\mathrm{d} S_{1}(t) \geq & \left(D\left(S_{1}^{0}-S_{1}(t)\right)-\frac{r_{1} S_{1}(t) \varepsilon}{K_{1}+S_{1}(t)}\right) \mathrm{d} t \\
& -\frac{\sigma_{1} S_{1}(t) \varepsilon \mathrm{d} B(t)}{K_{1}+S_{1}(t)} \\
\frac{\mathrm{d} S_{1}(t)}{\mathrm{d} t} \geq & D S_{1}^{0}-\left(D+\frac{r_{1} \varepsilon}{K_{1}}+\frac{\sigma_{1} \varepsilon|B(t)|}{K_{1}}\right) S_{1}(t) .
\end{aligned}
$$

Then when $\varepsilon \rightarrow 0$ we have

$$
\liminf _{t \rightarrow+\infty} S_{1}(t) \geq S_{1}^{0} .
$$

On the other hand from the proof of Lemma 2, we have

$$
\lim _{t \rightarrow+\infty} S_{1}(t) \leq S_{1}^{0}+\varepsilon
$$

Let $\varepsilon \rightarrow 0$. Then one has

$$
\limsup _{t \rightarrow+\infty} S_{1}(t) \leq S_{1}^{0}
$$

From (47) and (49), we have

$$
\lim _{t \rightarrow+\infty} S_{1}(t)=S_{1}^{0}
$$

almost surely. that

By employing the method similar above, it then follows

$$
\lim _{t \rightarrow+\infty} S_{2}(t)=S_{2}^{0}
$$

almost surely. This completes the proof of Theorem 6 .

\subsection{Permanence in Mean}

Theorem 7. If $\mathscr{R}>1$, then the microorganism $X(t)$ is permanent in mean; moreover, $X(t)$ satisfies

$$
\liminf _{t \rightarrow+\infty}\langle X(t)\rangle \geq D \Delta\left(\mathscr{R}^{*}-1\right),
$$

where $\Delta=\min \left\{\left(K_{1}+S_{1}^{0}\right) D / r_{1},\left(K_{2}+S_{2}^{0}\right) D / r_{2}\right\}$.

Proof. Integrating from 0 to $t$ and dividing by $t$ on both sides of system (5) yield

$$
\begin{aligned}
\Theta(t) \triangleq & \frac{S_{1}(t)-S_{1}(0)}{t}+\frac{S_{2}(t)-S_{2}(0)}{t} \\
& +\frac{X(t)-X(0)}{t} \\
= & D\left(S_{1}^{0}+S_{2}^{0}\right)-D\left(\left\langle S_{1}(t)\right\rangle+\left\langle S_{2}(t)\right\rangle\right) \\
& -D\langle X(t)\rangle .
\end{aligned}
$$

Then one can get

$$
\begin{aligned}
D\langle X(t)\rangle= & D\left(S_{1}^{0}+S_{2}^{0}\right)-D\left(\left\langle S_{1}(t)\right\rangle+\left\langle S_{2}(t)\right\rangle\right) \\
& -\Theta(t) .
\end{aligned}
$$

Applying Itô’s formula gives

$$
\begin{aligned}
& \mathrm{d} \ln X(t)=\left(r_{1} \phi_{1}(t)+r_{2} \phi_{2}(t)-D-\frac{\sigma_{1}^{2}}{2} \phi_{1}^{2}(t)\right. \\
& \left.-\frac{\sigma_{2}^{2}}{2} \phi_{2}^{2}(t)\right) \mathrm{d} t+\frac{\sigma_{1} S_{1}(t) \mathrm{d} B(t)}{K_{1}+S_{1}(t)} \\
& +\frac{\sigma_{2} S_{2}(t) \mathrm{d} B(t)}{K_{2}+S_{2}(t)} \\
& \geq\left(r_{1} \phi_{1}(t)+r_{2} \phi_{2}(t)-D-\frac{\sigma_{1}^{2}}{2}\left(\frac{S_{1}^{0}}{K_{1}+S_{1}^{0}}\right)^{2}\right. \\
& \left.-\frac{\sigma_{2}^{2}}{2}\left(\frac{S_{2}^{0}}{K_{2}+S_{2}^{0}}\right)^{2}\right) \mathrm{d} t+\frac{\sigma_{1} S_{1}(t) \mathrm{d} B(t)}{K_{1}+S_{1}(t)} \\
& +\frac{\sigma_{2} S_{2}(t) \mathrm{d} B(t)}{K_{2}+S_{2}(t)} .
\end{aligned}
$$

Integrating from 0 to $t$ and dividing by $t$ on both sides of (55) yields

$$
\begin{aligned}
& \frac{\ln X(t)-\ln X(0)}{t} \\
& \geq r_{1} \frac{1}{t} \int_{0}^{t} \phi_{1}(\theta) \mathrm{d} \theta+r_{2} \frac{1}{t} \int_{0}^{t} \phi_{2}(\theta) \mathrm{d} \theta \\
& \quad-\left[D+\frac{\sigma_{1}^{2}}{2}\left(\frac{S_{1}^{0}}{K_{1}+S_{1}^{0}}\right)^{2}+\frac{\sigma_{2}^{2}}{2}\left(\frac{S_{2}^{0}}{K_{2}+S_{2}^{0}}\right)^{2}\right] \\
& \quad+\frac{M_{1}(t)}{t}+\frac{M_{2}(t)}{t},
\end{aligned}
$$

where $M_{1}(t)=\int_{0}^{t}\left(\sigma_{1} S_{1}(\tau) /\left(K_{1}+S_{1}(\tau)\right)\right) \mathrm{d} B(\tau), M_{2}(t)=$ $\int_{0}^{t}\left(\sigma_{2} S_{2}(\tau) /\left(K_{2}+S_{2}(\tau)\right)\right) \mathrm{d} B(\tau)$. Noticing that

$$
\begin{aligned}
& \int_{0}^{t} \phi_{1}(\theta) \mathrm{d} \theta=\int_{0}^{t} \frac{S_{1}(\theta)}{K_{1}+S_{1}(\theta)} \mathrm{d} \theta \geq \int_{0}^{t} \frac{S_{1}(\theta)}{K_{1}+S_{1}^{0}} \mathrm{~d} \theta, \\
& \int_{0}^{t} \phi_{2}(\theta) \mathrm{d} \theta=\int_{0}^{t} \frac{S_{2}(\theta)}{K_{2}+S_{2}(\theta)} \mathrm{d} \theta \geq \int_{0}^{t} \frac{S_{2}(\theta)}{K_{2}+S_{2}^{0}} \mathrm{~d} \theta,
\end{aligned}
$$


then we have

$$
\begin{aligned}
& \frac{\ln X(t)-\ln X(0)}{t} \\
& \geq-\left[D+\frac{\sigma_{1}^{2}}{2}\left(\frac{S_{1}^{0}}{K_{1}+S_{1}^{0}}\right)^{2}+\frac{\sigma_{2}^{2}}{2}\left(\frac{S_{2}^{0}}{K_{2}+S_{2}^{0}}\right)^{2}\right] \\
& +\frac{r_{1}}{K_{1}+S_{1}^{0}}\left\langle S_{1}(t)\right\rangle+\frac{r_{2}}{K_{2}+S_{2}^{0}}\left\langle S_{2}(t)\right\rangle+\frac{M_{1}(t)}{t} \\
& +\frac{M_{2}(t)}{t} \\
& =-\left[D+\frac{\sigma_{1}^{2}}{2}\left(\frac{S_{1}^{0}}{K_{1}+S_{1}^{0}}\right)^{2}+\frac{\sigma_{2}^{2}}{2}\left(\frac{S_{2}^{0}}{K_{2}+S_{2}^{0}}\right)^{2}\right] \\
& +\frac{r_{2}}{\left(K_{2}+S_{2}^{0}\right)}\left(S_{1}^{0}+S_{2}^{0}\right) \\
& +\left(\frac{r_{1}}{K_{1}+S_{1}^{0}}-\frac{r_{2}}{K_{2}+S_{2}^{0}}\right)\left\langle S_{1}(t)\right\rangle \\
& -\frac{r_{2}}{K_{2}+S_{2}^{0}}\langle X(t)\rangle-\frac{r_{2}}{K_{2}+S_{2}^{0}} \frac{\Theta(t)}{D}+\frac{M_{1}(t)}{t} \\
& +\frac{M_{2}(t)}{t}
\end{aligned}
$$

If $r_{1} /\left(K_{1}+S_{1}^{0}\right) \leq r_{2} /\left(K_{2}+S_{2}^{0}\right)$, we can get

$$
\begin{aligned}
\frac{\ln X(t)-\ln X(0)}{t} & -\left[D+\frac{\sigma_{1}^{2}}{2}\left(\frac{S_{1}^{0}}{K_{1}+S_{1}^{0}}\right)^{2}+\frac{\sigma_{2}^{2}}{2}\left(\frac{S_{2}^{0}}{K_{2}+S_{2}^{0}}\right)^{2}\right] \\
& +\frac{r_{2}}{\left(K_{2}+S_{2}^{0}\right)}\left(S_{1}^{0}+S_{2}^{0}\right) \\
& +\left(\frac{r_{1}}{K_{1}+S_{1}^{0}}-\frac{r_{2}}{K_{2}+S_{2}^{0}}\right) S_{1}^{0}-\frac{r_{2}}{K_{2}+S_{2}^{0}}\langle X(t)\rangle \\
& -\frac{r_{2}}{K_{2}+S_{2}^{0}} \frac{\Theta(t)}{D}+\frac{M_{1}(t)}{t}+\frac{M_{2}(t)}{t} \\
\geq & -\left[D+\frac{\sigma_{1}^{2}}{2}\left(\frac{S_{1}^{0}}{K_{1}+S_{1}^{0}}\right)^{2}+\frac{\sigma_{2}^{2}}{2}\left(\frac{S_{2}^{0}}{K_{2}+S_{2}^{0}}\right)^{2}\right] \\
& +\frac{r_{2} S_{2}^{0}}{K_{2}+S_{2}^{0}}+\frac{r_{1} S_{1}^{0}}{K_{1}+S_{1}^{0}}-\frac{r_{2}}{K_{2}+S_{2}^{0}}\langle X(t)\rangle \\
& -\frac{r_{2}}{K_{2}+S_{2}^{0}} \frac{\Theta(t)}{D}+\frac{M_{1}(t)}{t}+\frac{M_{2}(t)}{t} .
\end{aligned}
$$

By inequality (59), we have

$$
\begin{aligned}
& \langle X(t)\rangle \geq \frac{K_{2}+S_{2}^{0}}{r_{2}}\left(\frac{r_{1} S_{1}^{0}}{K_{1}+S_{1}^{0}}+\frac{r_{2} S_{2}^{0}}{K_{2}+S_{2}^{0}}\right. \\
& \left.-\frac{\sigma_{1}^{2}}{2}\left(\frac{S_{1}^{0}}{K_{1}+S_{1}^{0}}\right)^{2}-\frac{\sigma_{2}^{2}}{2}\left(\frac{S_{2}^{0}}{K_{2}+S_{2}^{0}}\right)^{2}-D\right) \\
& -\frac{K_{2}+S_{2}^{0}}{r_{2}}\left(\frac{\ln X(t)-\ln X(0)}{t}+\frac{r_{2}}{K_{2}+S_{2}^{0}} \frac{\Theta(t)}{D}\right. \\
& \left.+\frac{M_{1}(t)}{t}+\frac{M_{2}(t)}{t}\right) .
\end{aligned}
$$

By Lemma 3, we get that $\lim _{t \rightarrow+\infty}(M(t) / t)=0$. According to Lemma 2, one sees that $\lim \sup _{t \rightarrow+\infty} S_{1}(t) \leq S_{1}^{0}$, $\lim \sup _{t \rightarrow+\infty} S_{2}(t) \leq S_{2}^{0}$, and $\lim \sup _{t \rightarrow+\infty} X(t) \leq C_{0}+S_{2}^{0}$, and then one has $\lim _{t \rightarrow+\infty}(\ln X(t) / t)=0$ and $\lim _{t \rightarrow+\infty} \Theta(t)=0$. Thus taking the inferior limit of both sides of (60) yields

$$
\liminf _{t \rightarrow+\infty}\langle X(t)\rangle \geq \frac{\left(K_{2}+S_{2}^{0}\right) D}{r_{2}}\left(\mathscr{R}^{*}-1\right)
$$

And if $r_{1} /\left(K_{1}+S_{1}^{0}\right) \geq r_{2} /\left(K_{2}+S_{2}^{0}\right)$, we can get

$$
\begin{aligned}
& \frac{\ln X(t)-\ln X(0)}{t} \geq-\left[D+\frac{\sigma_{1}^{2}}{2}\left(\frac{S_{1}^{0}}{K_{1}+S_{1}^{0}}\right)^{2}\right. \\
& \left.+\frac{\sigma_{2}^{2}}{2}\left(\frac{S_{2}^{0}}{K_{2}+S_{2}^{0}}\right)^{2}\right]+\frac{r_{1}}{K_{1}+S_{1}^{0}}\left(\left(S_{1}^{0}+S_{2}^{0}\right)\right. \\
& \left.-\left\langle S_{2}(t)\right\rangle-\langle X(t)\rangle-\frac{\Theta(t)}{D}\right)+\frac{r_{2}}{K_{2}+S_{2}^{0}}\left\langle S_{2}(t)\right\rangle \\
& +\frac{M_{1}(t)}{t}+\frac{M_{2}(t)}{t}=-\left[D+\frac{\sigma_{1}^{2}}{2}\left(\frac{S_{1}^{0}}{K_{1}+S_{1}^{0}}\right)^{2}\right. \\
& \left.+\frac{\sigma_{2}^{2}}{2}\left(\frac{S_{2}^{0}}{K_{2}+S_{2}^{0}}\right)^{2}\right]+\frac{r_{1} S_{1}^{0}}{K_{1}+S_{1}^{0}}+\frac{r_{2} S_{2}^{0}}{K_{2}+S_{2}^{0}} \\
& +\frac{r_{1}}{K_{1}+S_{1}^{0}}\langle X(t)\rangle-\frac{r_{2}}{K_{2}+S_{2}^{0}} \frac{\Theta(t)}{D}+\frac{M_{1}(t)}{t} \\
& +\frac{M_{2}(t)}{t},
\end{aligned}
$$

where $M_{1}(t)=\int_{0}^{t}\left(\sigma_{1} S_{1}(\tau) /\left(K_{1}+S_{1}(\tau)\right)\right) \mathrm{d} B(\tau), M_{2}(t)=$ $\int_{0}^{t}\left(\sigma_{2} S_{2}(\tau) /\left(K_{2}+S_{2}(\tau)\right)\right) \mathrm{d} B(\tau)$. 
Inequality (62) can be rewritten as

$$
\begin{aligned}
& \langle X(t)\rangle \geq \frac{\left(K_{2}+S_{2}^{0}\right)}{r_{2}}\left(\frac{r_{1} S_{1}^{0}}{K_{1}+S_{1}^{0}}+\frac{r_{2} S_{2}^{0}}{K_{2}+S_{2}^{0}}\right. \\
& \left.-\frac{\sigma_{1}^{2}}{2}\left(\frac{S_{1}^{0}}{K_{1}+S_{1}^{0}}\right)^{2}-\frac{\sigma_{2}^{2}}{2}\left(\frac{S_{2}^{0}}{K_{2}+S_{2}^{0}}\right)^{2}-D\right) \\
& -\frac{\left(K_{2}+S_{2}^{0}\right)}{r_{2}}\left(\frac{\ln X(t)-\ln X(0)}{t}+\frac{r_{1}}{K_{1}+S_{1}^{0}} \frac{\Theta(t)}{D}\right. \\
& \left.+\frac{M_{1}(t)}{t}+\frac{M_{2}(t)}{t}\right) .
\end{aligned}
$$

Taking the inferior limit of both sides of (63) yields

$$
\liminf _{t \rightarrow+\infty}\langle X(t)\rangle \geq \frac{\left(K_{1}+S_{1}^{0}\right) D}{r_{1}}\left(\mathscr{R}^{*}-1\right) .
$$

Let $\Delta=\min \left\{\left(K_{1}+S_{1}^{0}\right) D / r_{1},\left(K_{2}+S_{2}^{0}\right) D / r_{2}\right\}$, and we get from (61) and (64)

$$
\liminf _{t \rightarrow+\infty}\langle X(t)\rangle \geq D \Delta\left(\mathscr{R}^{*}-1\right) .
$$

This completes the proof of Theorem 7.

Remark 8. Theorems 6 and 7 show that the condition for the microorganism to go to extinction or permanence depends on the intensity of the noise disturbances completely. And small noise disturbances will be beneficial to the cultivation of the microorganism; conversely, large white noise disturbance is harmful to the cultivation of the microorganism.

\section{Conclusion and Numerical Simulation}

This paper proposes and investigates a new stochastic chemostat model with two substitutable nutrients and one microorganism. Then main objective in this paper is to investigate the threshold dynamics of stochastic chemostat model (5) and explore the conditions which can determine the extinction and permanence of the microorganism using two substitutable nutrients. Firstly, for the corresponding deterministic model, the threshold for extinction or existence of the microorganism is obtained by analyzing the stability of the equilibria. Then the threshold of the stochastic chemostat for the extinction and the permanence in mean of the microorganism is explored. The results show that there exists a significant difference between the threshold of the deterministic system and the stochastic system, which makes the persistent microorganism of a deterministic system become extinct due to large stochastic disturbance. That is, large stochastic disturbance is harmful to the cultivation of the

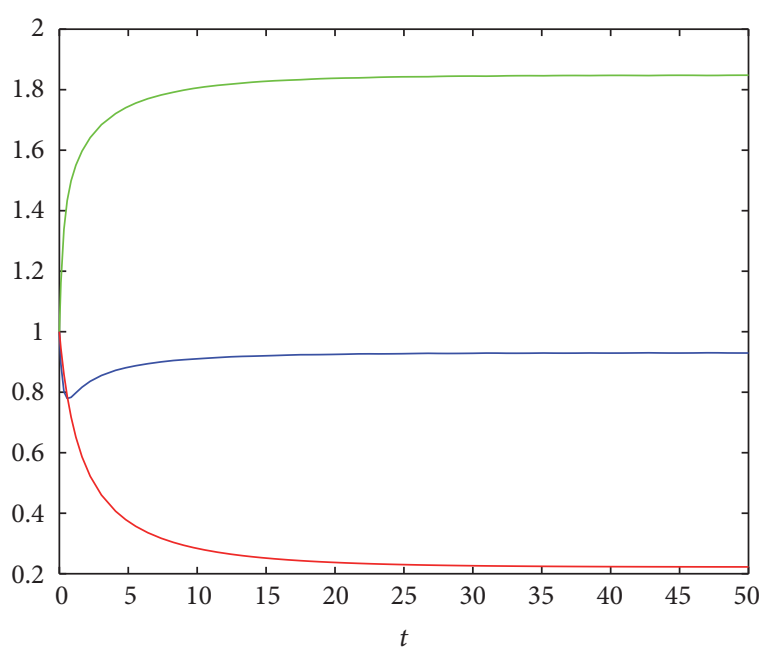

$S_{1}(t)$ of deterministic system
$S_{2}(t)$ of deterministic system

FIgure 1: Time series for the paths $S_{1}(t), S_{2}(t), X(t)$ for the deterministic system with $D=3.8, S_{1}^{0}=1, S_{2}^{0}=2, r_{1}=2.5, r_{2}=4$, $K_{1}=1, K_{2}=1, \mathscr{R}=1.0307>1$.

microorganism. It is worth mentioning that this paper is a promotion of the work of $\mathrm{Xu}$ and Yuan [35].

Next, using the Euler Maruyama (EM) method [40], we give some numerical simulation to illustrate the extinction and persistence of the microorganism in stochastic system and corresponding deterministic system for comparison.

Firstly, we begin from a deterministic system; the basic parameters are set as $D=3.8, S_{1}^{0}=1, S_{2}^{0}=2$, $r_{1}=2.5, r_{2}=4, K_{1}=1$, and $K_{2}=1$. Direct calculation shows that $\delta_{1}=0.8, \delta_{2}=0.375$, and $\mathscr{R}=$ $1.0307>1$. Then according to Theorems 4 and 5 , the deterministic system has a unique stable positive equilibrium $E^{*}(0.9295785859,1.848231322,0.2221900926)$, which is locally stable and the deterministic system is permanent (see Figure 1).

Next, we consider the influence of stochastic disturbance on the above deterministic system. According to Theorem 6, different parameters are chosen to give insights into the reasonability of the results stated in Theorem 6 .

We choose different value of parameters $\sigma_{1}$ and $\sigma_{2}$ and discuss below five different cases.

Case 1. Choose $\sigma_{1}=1.6, \sigma_{2}=1.8$, by direct calculation; we have $r_{1}^{2} / 2 \sigma_{1}^{2}+r_{2}^{2} / 2 \sigma_{2}^{2}=3.6898<D=3.8$. Then, by Theorem 6 , the microorganism eventually tends to be extinct (see Figure 2(a)).

Case 2. Choose $\sigma_{1}=1, \sigma_{2}=0.5$, by direct calculation; we have $r_{1}^{2} / 2 \sigma_{1}^{2}+r_{2} S_{2}^{0} /\left(K_{2}+S_{2}^{0}\right)-\left(\sigma_{2}^{2} / 2\right)\left(S_{2}^{0} /\left(K_{2}+S_{2}^{0}\right)\right)^{2}=0.5139<$ $D=3.8$. Then, by Theorem 6 , the microorganism eventually tends to be extinct (see Figure 2(b)). 


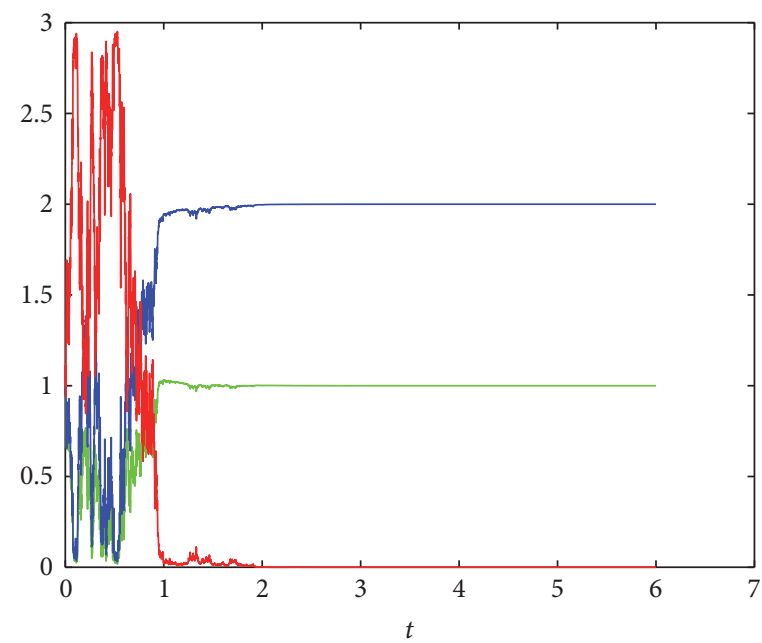

- $S_{1}(t)$ of stochastic system

- $S_{2}(t)$ of stochastic system

- $X(t)$ of stochastic system

(a) $\sigma_{1}=1.6, \sigma_{2}=1.8$

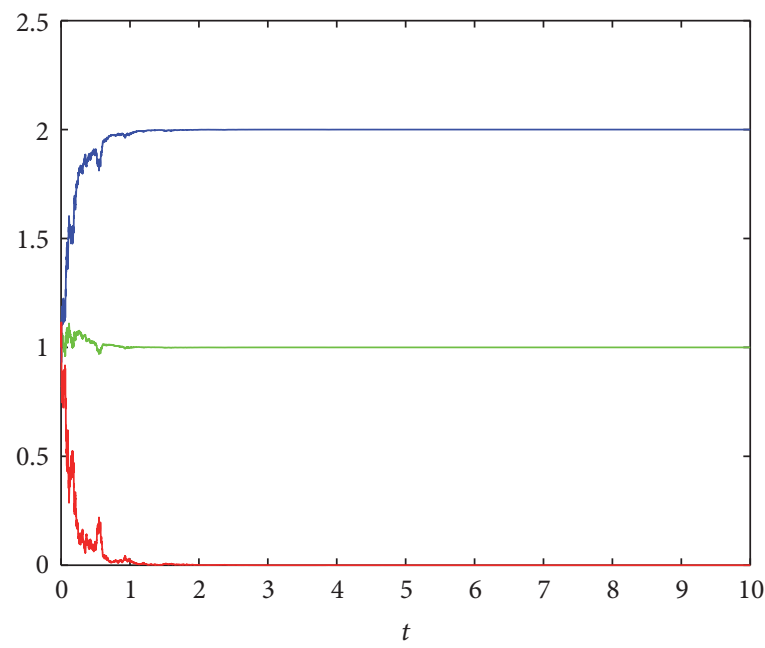

$S_{1}(t)$ of stochastic system

- $S_{2}(t)$ of stochastic system

- $X(t)$ of stochastic system

(c) $\sigma_{1}=0.7, \sigma_{2}=1.3$

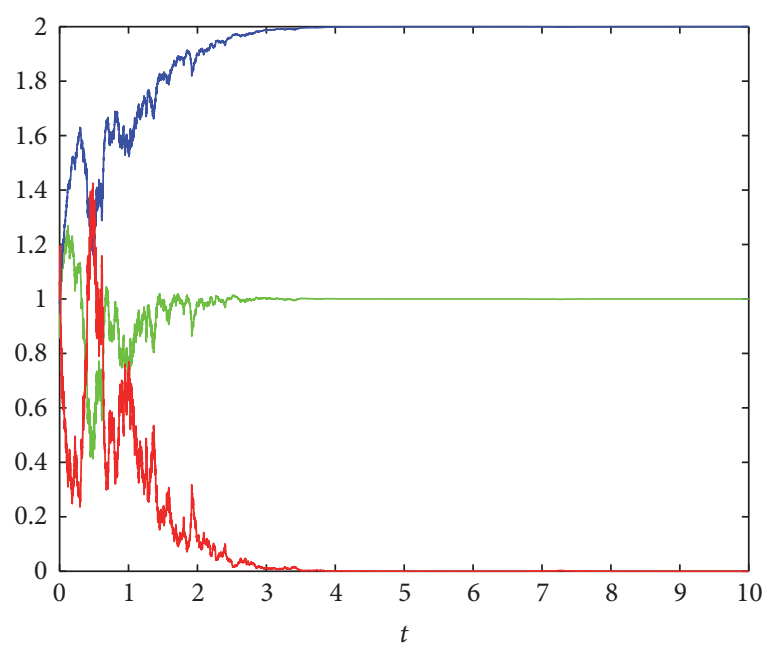

$S_{1}(t)$ of stochastic system

$-S_{2}(t)$ of stochastic system

- $X(t)$ of stochastic system

(b) $\sigma_{1}=0.7, \sigma_{2}=1.3$

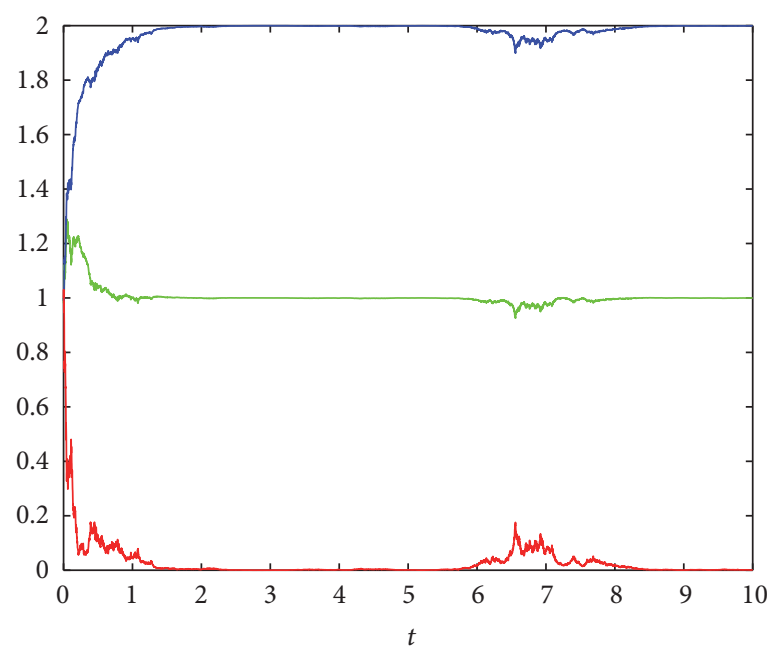

$S_{1}(t)$ of stochastic system

- $S_{2}(t)$ of stochastic system

- $X(t)$ of stochastic system

(d) $\sigma_{1}=0.7, \sigma_{2}=0.6, \mathscr{R}^{\prime}=0.9935<1$

FIGURE 2: Time series for the paths $S_{1}(t), S_{2}(t), X(t)$ for the stochastic system.

Case 3. Choose $\sigma_{1}=0.7, \sigma_{2}=1.3$, by direct calculation; we have $r_{2}^{2} / 2 \sigma_{2}^{2}+r_{1} S_{1}^{0} /\left(K_{1}+S_{1}^{0}\right)-\left(\sigma_{1}^{2} / 2\right)\left(S_{1}^{0} /\left(K_{1}+S_{1}^{0}\right)\right)^{2}=3.5450<$ $D=3.8$. Then, by Theorem 6 , the microorganism eventually tends to be extinct (see Figure 2(c)).

Case 4. Choose $\sigma_{1}=0.7, \sigma_{2}=0.6$, by direct calculation; we have $\mathscr{R}^{\prime}=0.9935<1$. Then, by Theorem 6 , the microorganism eventually tends to be extinct (see Figure 2(d)).

Case 5. Choose $\sigma_{1}=0.2, \sigma_{2}=0.3$, by direct calculation; we have $\mathscr{R}^{\prime}=1.0241>1$. Then, by Theorem 7, the microorganism is persistent (see Figure 3).

\section{Conflicts of Interest}

The authors declare that there are no conflicts of interest regarding the publication of this paper.

\section{Acknowledgments}

This work is supported by Shandong Provincial Natural Science Foundation (no. ZR2015AQ001), the National Natural Science Foundation of China (no. 11371230), and Research Funds for Joint Innovative Center for Safe and Effective 


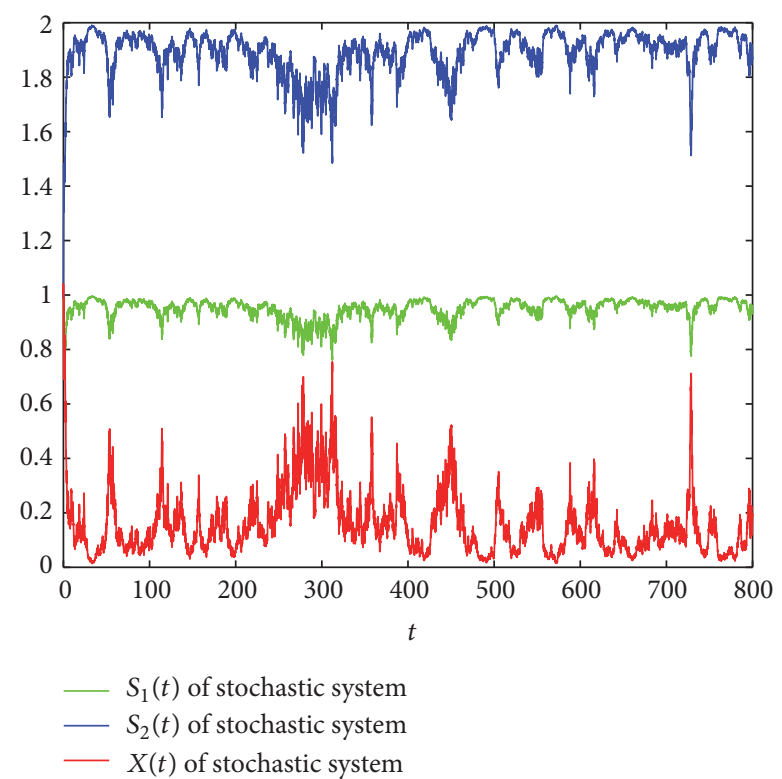

FIGURE 3: Time series for the paths $S_{1}(t), S_{2}(t), X(t)$ for the stochastic system with $\sigma_{1}=0.2, \sigma_{2}=0.3, \mathscr{R}^{\prime}=1.0241>1$.

Mining Technology and Equipment of Coal Resources by Shandong Province and SDUST (2014TDJH102).

\section{References}

[1] D. Herbert, R. Elsworth, and R. C. Telling, "The continuous culture of bacteria; a theoretical and experimental study," Journal of General Microbiology, vol. 14, no. 3, pp. 601-622, 1956.

[2] H. L. Smith and P. Waltman, The Theory of the Chemostat: Dynamics of Microbial Competition, Cambridge Studies in Mathematical Biology, Cambridge University Press, Cambridge, UK, 1995.

[3] T. Zhang, T. Zhang, and X. Meng, "Stability analysis of a chemostat model with maintenance energy," Applied Mathematics Letters. An International Journal of Rapid Publication, vol. 68, pp. 1-7, 2017.

[4] X. Meng, L. Wang, and T. Zhang, "Global dynamics analysis of a nonlinear impulsive stochastic chemostat system in a polluted environment," Journal of Applied Analysis and Computation, vol. 6, no. 3, pp. 865-875, 2016.

[5] W. Wang, W. Ma, and H. Yan, "Global dynamics of modeling flocculation of microorganism," Applied Sciences (Switzerland), vol. 6 , no. 8 , article no. 221, 2016.

[6] X. Meng, Q. Gao, and Z. Li, "The effects of delayed growth response on the dynamic behaviors of the Monod type chemostat model with impulsive input nutrient concentration," Nonlinear Analysis. Real World Applications. An International Multidisciplinary Journal, vol. 11, no. 5, pp. 4476-4486, 2010.

[7] J. Gao, B. Shen, E. Feng, and Z. Xiu, "Modelling and optimal control for an impulsive dynamical system in microbial fedbatch culture," Computational and Applied Mathematics, vol. 32, no. 2, pp. 275-290, 2013.

[8] T. Zhang, W. Ma, and X. Meng, "Global dynamics of a delayed chemostat model with harvest by impulsive flocculant input," Advances in Difference Equations, Paper No. 115, 17 pages, 2017.
[9] J. Monod, Recherches Sur La Croissance Des Cultures Bacteriennes, Hermann, Paris, France, 1942.

[10] M. M. Ballyk and G. S. Wolkowicz, "An examination of the thresholds of enrichment: a resource-based growth model," Journal of Mathematical Biology, vol. 33, no. 4, pp. 435-457, 1995.

[11] B. Li, G. S. Wolkowicz, and Y. Kuang, "Global asymptotic behavior of a chemostat model with two perfectly complementary resources and distributed delay," SIAM Journal on Applied Mathematics, vol. 60, no. 6, pp. 2058-2086, 2000.

[12] J. A. Leon and D. B. Tumpson, "Competition between two species for two complementary or substitutable resources," Journal of Theoretical Biology, vol. 50, no. 1, pp. 185-201, 1975.

[13] W. Harder and L. Dijkhuizen, "Strategies of mixed substrate utilization in microorganisms," Philosophical transactions of the Royal Society of London. Series B: Biological sciences, vol. 297, no. 1088, pp. 459-480, 1982.

[14] J. P. Braselton, M. L. Abell, and L. M. Braselton, "Comparing the effects of interactive and noninteractive complementary nutrients on growth in a chemostat," Open Journal of Applied Sciences, vol. 3, no. 05, pp. 323-331, 2013.

[15] A. Miao, J. Zhang, T. Zhang, and B. . Pradeep, "Threshold Dynamics of a Stochastic SIR Model with Vertical Transmission and VACcination," Computational and Mathematical Methods in Medicine, Art. ID 4820183, 10 pages, 2017.

[16] A. Miao, X. Wang, T. Zhang, W. Wang, and B. Sampath Aruna Pradeep, "Dynamical analysis of a stochastic SIS epidemic model with nonlinear incidence rate and double epidemic hypothesis," Advances in Difference Equations, 2017:226 pages, 2017.

[17] S. Zhang, X. Meng, T. Feng, and T. Zhang, "Dynamics analysis and numerical simulations of a stochastic non-autonomous predator-prey system with impulsive effects," Nonlinear Analysis: Hybrid Systems, 2017.

[18] Y. Wang and Z. Huang, "Backward stochastic differential equations with non-Lipschitz coefficients," Statistics and Probability Letters, vol. 79, no. 12, pp. 1438-1443, 2009.

[19] T. Feng, X. Meng, L. Liu, and S. Gao, "Application of inequalities technique to dynamics analysis of a stochastic ecoepidemiology model," Journal of Inequalities and Applications, Paper No. 327, 29 pages, 2016.

[20] H. Ma and Y. Jia, "Stability analysis for stochastic differential equations with infinite Markovian switchings," Journal of Mathematical Analysis and Applications, vol. 435, no. 1, pp. 593-605, 2016.

[21] X. Meng, S. Zhao, T. Feng, and T. Zhang, "Dynamics of a novel nonlinear stochastic SIS epidemic model with double epidemic hypothesis," Journal of Mathematical Analysis and Applications, vol. 433, no. 1, pp. 227-242, 2016.

[22] Y. Zhao and W. Zhang, "Observer-based controller design for singular stochastic Markov jump systems with state dependent noise," Journal of Systems Science and Complexity, vol. 29, no. 4, pp. 946-958, 2016.

[23] X.-z. Meng, "Stability of a novel stochastic epidemic model with double epidemic hypothesis," Applied Mathematics and Computation, vol. 217, no. 2, pp. 506-515, 2010.

[24] W. Zhang, Y. Zhao, and L. Sheng, "Some remarks on stability of stochastic singular systems with state-dependent noise," Automatica. A Journal of IFAC, the International Federation of Automatic Control, vol. 51, pp. 273-277, 2015.

[25] C. Tan and W. Zhang, "On observability and detectability of continuous-time stochastic Markov jump systems," Journal of Systems Science and Complexity, vol. 28, no. 4, pp. 830-847, 2015. 
[26] X. Li, X. Lin, and Y. Lin, "Lyapunov-type conditions and stochastic differential equations driven by G-Brownian motion," Journal of Mathematical Analysis and Applications, vol. 439, no. 1, pp. 235-255, 2016.

[27] H.-j. Ma and T. Hou, "A separation theorem for stochastic singular linear quadratic control problem with partial information," Acta Mathematicae Applicatae Sinica. English Series, vol. 29, no. 2, pp. 303-314, 2013.

[28] W. Zhang, X. Lin, and B.-S. Chen, "LaSalle-type theorem and its applications to infinite horizon optimal control of discretetime nonlinear stochastic systems," Institute of Electrical and Electronics Engineers. Transactions on Automatic Control, vol. 62, no. 1, pp. 250-261, 2017.

[29] Z. Chang, X. Meng, and X. Lu, "Analysis of a novel stochastic SIRS epidemic model with two different saturated incidence rates," Physica A. Statistical Mechanics and its Applications, vol. 472, pp. 103-116, 2017.

[30] X. Liu, Y. Li, and W. Zhang, "Stochastic linear quadratic optimal control with constraint for discrete-time systems," Applied Mathematics and Computation, vol. 228, pp. 264-270, 2014.

[31] W. Zhao, J. Li, T. Zhang, X. Meng, and T. Zhang, "Persistence and ergodicity of plant disease model with Markov conversion and impulsive toxicant input," Communications in Nonlinear Science and Numerical Simulation, vol. 48, pp. 70-84, 2017.

[32] L. Liu and X. Meng, "Optimal harvesting control and dynamics of two-species stochastic model with delays," Advances in Difference Equations, Paper No. 18, 17 pages, 2017.

[33] X. Lin and R. Zhang, " $H_{\infty}$ control for stochastic systems with Poisson jumps," Journal of Systems Science and Complexity, vol. 24, no. 4, pp. 683-700, 2011.

[34] L. Imhof and S. Walcher, "Exclusion and persistence in deterministic and stochastic chemostat models," Journal of Differential Equations, vol. 217, no. 1, pp. 26-53, 2005.

[35] C. Xu and S. Yuan, "An analogue of break-even concentration in a simple stochastic chemostat model," Applied Mathematics Letters. An International Journal of Rapid Publication, vol. 48, pp. 62-68, 2015.

[36] F. Campillo, M. Joannides, and I. Larramendy-Valverde, "Stochastic modeling of the chemostat," Ecological Modelling, vol. 222, no. 15, pp. 2676-2689, 2011.

[37] C. Xu, S. Yuan, and T. Zhang, "Asymptotic behavior of a chemostat model with stochastic perturbation on the dilution rate," Abstract and Applied Analysis, vol. 2013, Article ID 423154, 2013.

[38] Z. Chen and T. Zhang, "Long time behaviour of a stochastic model for continuous flow bioreactor," Journal of Mathematical Chemistry, vol. 51, no. 2, pp. 451-464, 2013.

[39] D. Zhao and S. Yuan, "Critical result on the break-even concentration in a single-species stochastic chemostat model," Journal of Mathematical Analysis and Applications, vol. 434, no. 2, pp. 1336-1345, 2016.

[40] X. Mao, Stochastic Differential Equations and Applications, Elsevier, 2007. 


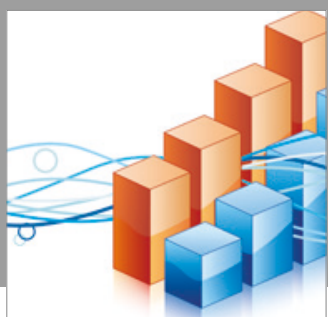

Advances in

Operations Research

vatersals

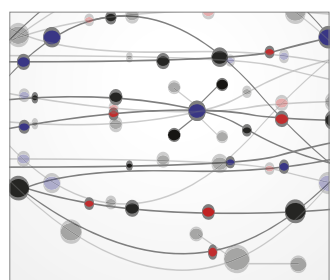

\section{The Scientific} World Journal
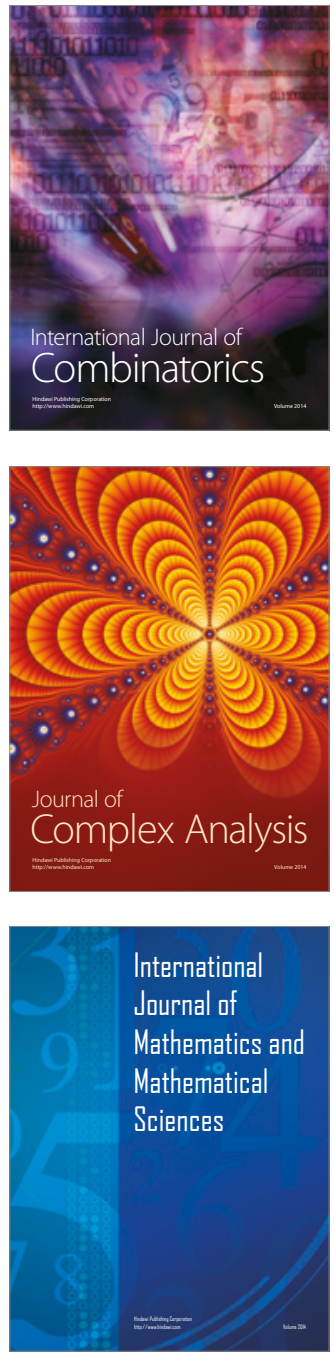
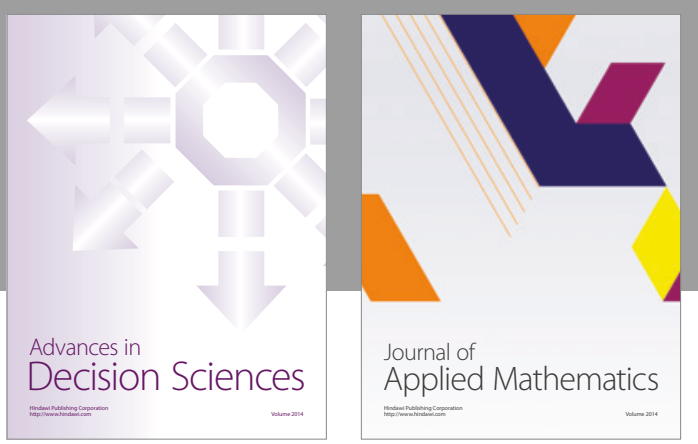

Algebra

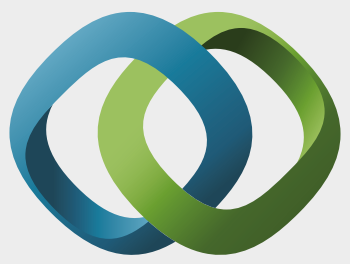

\section{Hindawi}

Submit your manuscripts at

https://www.hindawi.com
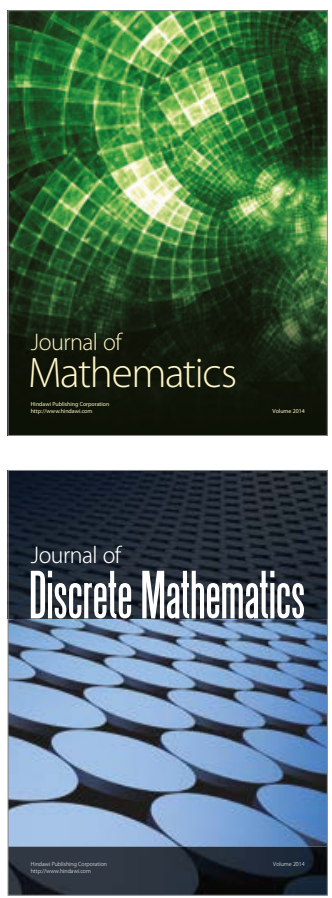

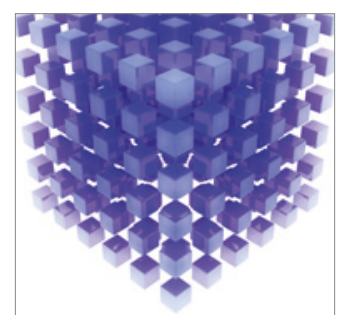

Mathematical Problems in Engineering
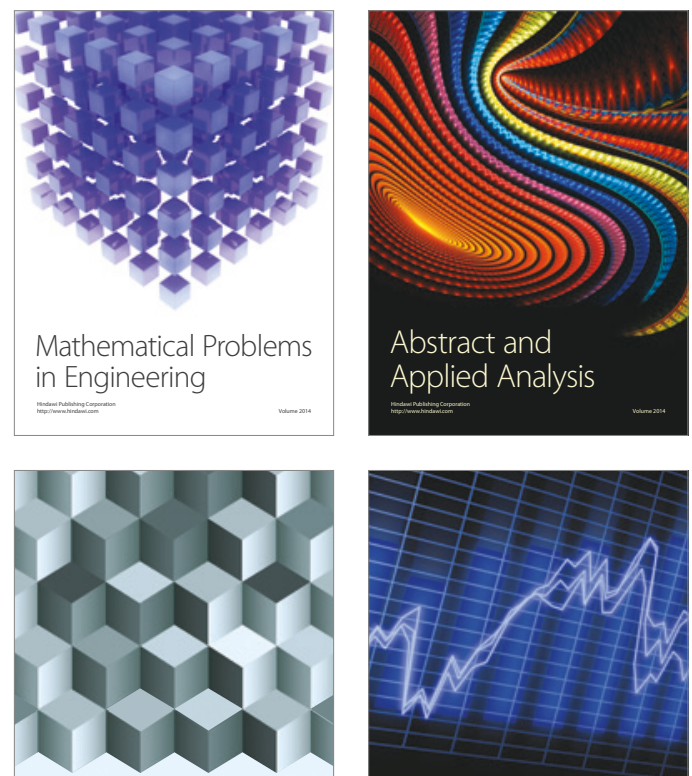

Journal of

Function Spaces

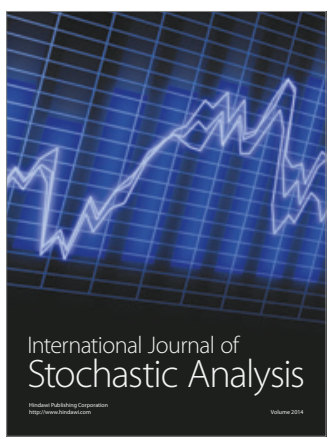

Probability and Statistics
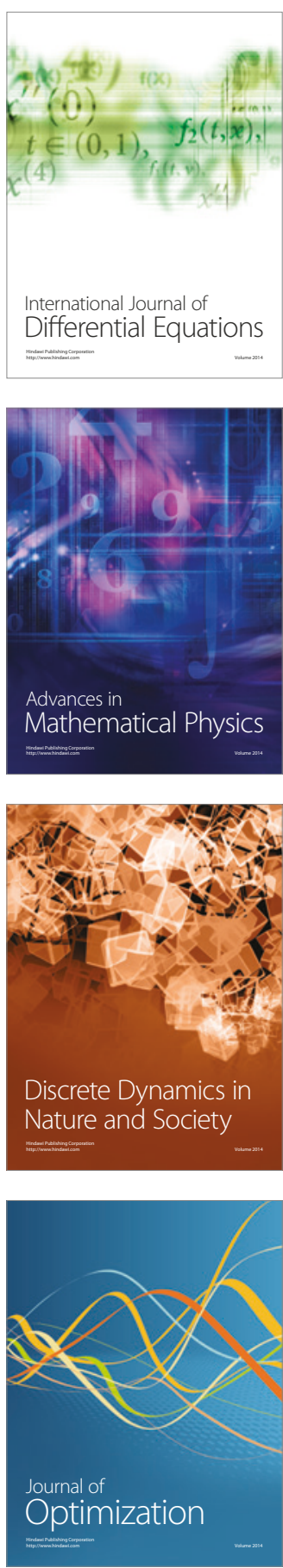